\title{
Rocky Saenz: Top 3 differentials in gastrointestinal imaging: a case review
}

\author{
Kristina Flicek ${ }^{1}$
}

Published online: 28 October 2019

(c) Springer Science+Business Media, LLC, part of Springer Nature 2019

This is a book review of the "Top 3 Differentials in Gastrointestinal Imaging: a case review" by Rocky C. Saenz. Per the preface, this book was aimed at radiology residents as well as other surgical or GI residents, fellows, and practicing physicians as both a resource for boards study or for use in daily practice.

This case-based textbook breaks down the GI system into five parts: hepatobiliary, pancreas/spleen, gastrointestinal track, mesentery/vascular, and abdominal wall/soft tissues. Given the breadth of this topic, this 300-page text is not all inclusive but rather a sample of commonly seen diagnoses. Each case has 1-4 pictures using most commonly a single modality (CT, fluoro, radiography, or MRI). Within each case, a short clinical history is given followed by key findings and a top 3 differential. Additional diagnostic considerations are also provided in a separate subsection followed by the diagnosis. Several diagnostic pearls are then provided for each case.

In concordance with the books targeted audience, I believe many of the cases are classic examples for residents and would be appropriate for first- through third-year residents. The material within the book may, however, be too simplistic and not in-depth enough for abdominal radiology fellows and practicing radiologists.

The positive aspects of this text include the concise matter in which the information is laid out. The different subsections were consistent throughout all the different cases, which made navigating the material easy.

There are several aspects in which this text could improve. First, many of the pictures are small, and the contrast of the pictures makes it difficult to appreciate the more subtle findings needed to arrive at the correct diagnosis. By moving the caption (currently located to the right of the image) below the image, there would be more space for larger or

Kristina Flicek

Flicek.Kristina@mayo.edu

1 Mayo Clinic Arizona, Scottsdale, AZ, USA more pictures to exemplify the case. Additionally I think that it would be helpful to group the cases with the same diagnosis together for easier comparison. For example, there are multiple examples of hepatic metastases; however, these are sprinkled throughout the hepatic chapter. By grouping them together, the reader would be better able to compare the subtle differences in the metastases as well as reinforce overarching themes.

In addition to these layout suggestions, there are several cases which are either inaccurate or misinterpreted. For example, in case 94, the caption states that there is a focal stricture within the sigmoid colon; however, the image clearly demonstrates a stricture within the transverse colon. In case 110 , the images are diagnostic of esophageal intramural pseudodiverticulosis. The diagnosis states that it is a case of esophageal candidiasis; however, imaging findings are not classic, and it is known that pseudo-diverticulosis can harbor Candida as a commensal organism. Several other examples of similar errors were identified.

As such, I believe that this case review book would benefit from significant layout improvements (most importantly picture size and contrast) as well as content editing to ensure that the content provided is accurate and not misleading to trainees.

\section{Compliance with ethical standards}

Conflicts of interest The author has no ethical or compliance issues concerning the submitted book review.

Publisher's Note Springer Nature remains neutral with regard to jurisdictional claims in published maps and institutional affiliations. 